\title{
Clinical value of circulating liver cancer cells for the diagnosis of hepatocellular carcinoma: A meta-analysis
}

\author{
TAO JIN ${ }^{1}$, HAO PENG ${ }^{1}$ and HONGYAN WU ${ }^{2}$ \\ ${ }^{1}$ Department of Orthopedics, Renmin Hospital of Three Gorges University, Yichang, Hubei 443000; \\ ${ }^{2}$ Department of Immunology, Medical College of Three Gorges University, Yichang, Hubei 443002, P.R. China
}

Received April 22, 2013; Accepted July 05, 2013

DOI: 10.3892/br.2013.139

\begin{abstract}
This meta-analysis was conducted to evaluate the clinical value of circulating tumor cells (CTCs) for liver tumor size, clinical stage and metastasis. The clinical studies on CTCs and liver cancer were electronically and manually retrieved and Review Manager 5.1 software was used to evaluate the quality of the obtained studies, extract data and conduct a meta-analysis. A total of 5 studies including 535 liver cancer patients were identified. The results of the meta-analysis revealed that the odds ratio (OR) values of CTCs-positive rates between large and small tumor size, tumor stage I/II and III/IV, as well as metastatic and non-metastatic groups were 12.12 (95\% CI: 7.84-18.74), 0.10 (95\% CI: 0.07-0.16) and 0.09 (95\% CI: 0.05-0.15), respectively, which demonstrated a significant difference. In conclusion, the detection of CTCs in the peripheral blood was clearly associated with tumor size, clinical stage and metastasis.
\end{abstract}

\section{Introduction}

In 1869, Ashworth (1) was the first to identify circulating tumor cells (CTCs) in the peripheral blood of cancer patients. Subsequent studies revealed that CTCs are of predictive value regarding metastasis, recurrence and prognosis of melanoma, breast, pancreatic, colorectal and lung cancer. CTCs are also predictive of the patient response to antitumor treatment and may assist in developing a customized treatment plan (2-5).

Primary hepatocellular carcinoma is one of the malignant tumors that metastasize hematogenously. Previous studies demonstrated that liver cancer cells enter the circulation at an early stage and CTCs in the blood form a foremost condition leading to recurrence and metastasis following liver cancer surgery. Therefore, an effective method for the identification of CTCs in the blood and the investigation of their biological

Correspondence to: Dr Hongyan Wu, Department of Immunology, Medical College of Three Gorges University, 8 Daxue, Yichang, Hubei 443002, P.R. China

E-mail: wuhongyan@ctgu.edu.cn

Key words: circulating tumor cells, liver cancer, meta-analysis, hepatic neoplasm, diagnosis characteristics may promote the early diagnosis of liver cancer and prediction of early metastasis.

Therefore, CTCs, as a potential independent diagnostic index, have been extensively investigated. The diagnostic value of circulating liver cancer cells for liver cancer is currently under investigation worldwide. However, the detection of circulating liver cancer cells cannot be used as a conventional clinical screening approach due to the following reasons: i) the sample size is relatively small; ii) the inspection technology lacks standardization and automation and complicated sample preparation procedures are required, which may lead to significant differences among the results from different laboratories, or even from the same laboratory; and iii) different reagents and methods may exhibit different specificities and sensitivities (6).

Therefore, this meta-analysis aimed to collect studies conducted in China and other countries that focused on the diagnostic value of circulating liver cancer cells for liver cancer. We aimed to summarize and analyze the studies and combine the specific conditions of the related cases to create a sample library and, furthermore, discuss the effect of circulating liver cancer cells on the relevant indices of liver cancer, such as stage, size, metastasis, recurrence, prognosis, survival time and sensitivity to treatment from a statistical view point.

\section{Materials and methods}

Study inclusion and exclusion criteria. Inclusion criteria for the present study were: i) Patients with aggressive liver cancer at preliminary diagnosis; ii) patients with aggressive liver cancer with distinct pathological evidence and evaluated as aggressive through $\alpha$-fetoprotein measurements, contrast-enhanced ultrasonography, computed tomography (CT), or positron emission tomography-CT; and iii) complete data records on liver cancer and CTCs.

The exclusion criteria were as follows: i) non-cancer liver diseases, such as hepatitis or cirrhosis; ii) liver metastasis from other malignant tumors; iii) novel detection methods for CTCs; and iv) reviews of the literature.

Literature retrieval. Using 'liver cancer' and 'circulating tumor cells' as the keywords, 116 foreign studies published between 1983 and 2012 were retrieved from foreign language databases 
Table I. Studies included in the meta-analysis.

\begin{tabular}{|c|c|c|c|c|c|}
\hline Author & Year & Country & Liver cancer samples (n) & Control samples (n) & Refs. \\
\hline Liu & 2007 & China & 75 & 25 & (7) \\
\hline Zuo & 2008 & China & 56 & 30 & (8) \\
\hline $\mathrm{Yu}$ & 2011 & China & 126 & 0 & (9) \\
\hline $\mathrm{Xu}$ & 2010 & China & 235 & 57 & (10) \\
\hline Vona et al & 2004 & France & 43 & 69 & $(11)$ \\
\hline
\end{tabular}

such as PubMed, Springer Protocols and Web of Knowledge. Thirty-two studies published in China between 2002 and 2011 were retrieved from domestic databases, such as CCPD and VIP Information. In total, 148 studies were collected, excluding the duplicates. In addition, Chinese studies on circulating liver cancer cells and full-text references were manually retrieved. The related studies were further tracked with a search engine and, if necessary, document delivery service was employed for acquisition of the full text and related data.

Data abstraction. The related studies were screened according to the criteria mentioned above, the eligible studies were identified, the full text was carefully read and data were abstracted, including author, publication year, nationality, number of cases included in the study, number of CTCs-positive cases with tumor diameters $>5$ or $\leq 5 \mathrm{~cm}$, number of CTCs-positive cases with tumor stages I/II and III/IV and number of CTCs-positive cases with or without metastasis.

Data analysis and statistical methods. The abstracted data were subjected to meta-analysis by using Review Manager 5.1 software. The odds ratio (OR) was analyzed as an efficacy parameter, with the $95 \%$ confidence interval (CI) representing the variable. A two-sided $\mathrm{P}$-value of $<0.05$ was considered to indicate a statistically significant difference.

The statistical heterogeneity among the groups was analyzed by the $\mathrm{Q}$ test. In the case of statistical homogeneity among the groups $\left(\mathrm{P}>0.10\right.$ and $\left.\mathrm{I}^{2}<50 \%\right)$, the fixed-effects model was selected for analysis; in the case of statistical heterogeneity $\left(\mathrm{P}<0.10\right.$ and $\left.50 \%<\mathrm{I}^{2}<70 \%\right)$, the random-effects model was selected instead.

A funnel plot was drawn with the software for assessing the publication bias.

\section{Results}

Study retrieval results. A total of 136 references, excluding the duplicates, were electronically and manually retrieved from relevant databases. In total, 108 apparently relevant studies were rejected after reading the abstracts and 28 studies entered the next assessment process. Twenty-two studies were rejected after reading the abstracts due to reasons such as incomplete tumor data and incomplete data on CTC positivity. Ultimately, 5 clinical comparative studies published between 2004 and 2011 were included in the meta-analysis.

Study information. Five clinical controlled trials conducted on a total of 535 patients were included, with 4 trials having been completed in China and 1 trial in France. The study information is presented in Table I.

\section{Correlation analysis of the abstracted data}

Analysis of the correlation between CTCs level and tumor size. Among the included cases, 247 were classified in the group with a tumor diameter of $>5 \mathrm{~cm}$, whereas 288 were classified in the group with a tumor diameter of $\leq 5 \mathrm{~cm}$. The statistical results of CTCs-positive rate in the two groups are presented in Table II. Of the 5 clinical controlled trials, the $>5 \mathrm{~cm}$ group included 175 CTCs-positive cases, whereas the $\leq 5 \mathrm{~cm}$ group included 50 cases. The result of the heterogeneity test is shown in Fig. 1, wherein $\chi^{2}=6.44$, degree of freedom $(\mathrm{DOF})=4, \mathrm{P}=0.17$ and $\mathrm{I}^{2}=38 \%$; therefore, the fixed-effects model was applied. It was observed that the difference in CTCs-positive rate in the peripheral blood between the $>5$ and $\leq 5 \mathrm{~cm}$ groups was statistically significant $(\mathrm{OR}=12.12$, 95\% CI: 7.84-18.74 and $\mathrm{P}<0.00001)$. This finding demonstrated that the CTCs-positive rate was directly correlated with tumor size, i.e., the larger the tumor, the higher the CTCs-positive rate in the peripheral blood. The funnel plot demonstrated that the bilateral scatter-plot distribution was generally symmetrical, with no significant publication bias (Fig. 2).

Analysis of the correlation between CTCs level and tumor stage. Among the included cases, 258 were in the stage I/II group and 277 were in the stage III/IV group. The statistical results of the CTCs-positive rate in the two groups are presented in Table III. Of the 5 clinical controlled trials, the I/II group included 43 CTCs-positive cases, whereas the III/IV group included 182 cases. According to the results of the $\mathrm{Q}$ test, no significantly statistical heterogeneity was observed between the two groups $\left(\chi^{2}=1.11, \mathrm{DOF}=4, \mathrm{P}=0.89\right.$ and $\left.\mathrm{I}^{2}=0 \%\right)$; therefore, the fixed effects model was applied. The difference in CTCs-positive rates in the peripheral blood between the two groups was found to be statistically significant $(\mathrm{OR}=0.10$, 95\% CI: 0.07-0.16; $\mathrm{P}<0.00001$ ) (Fig. 3). The comparison results revealed that the CTCs-positive rate in the peripheral blood in the stage III/IV group was significantly higher compared to that in the stage I/II group. This finding indicates that the tumor stage was directly correlated with the presence of CTCs in the peripheral blood, i.e., the more advanced the stage, the higher the probability of CTCs detected in the peripheral blood. The funnel plot revealed no significant publication bias (Fig. 4).

Analysis of the correlation between CTCs level and tumor metastasis. Among the included cases, 330 were classified in the metastasis and 205 in the non-metastasis group. The 
Table II. Association of circulating tumor cells (CTCs) level with $>5$ and $\leq 5 \mathrm{~cm}$ tumor size groups.

\begin{tabular}{|c|c|c|c|c|c|c|}
\hline \multirow[b]{2}{*}{ Study } & \multirow[b]{2}{*}{ Year } & \multicolumn{2}{|c|}{$>5 \mathrm{~cm}$ group } & \multicolumn{2}{|c|}{$\leq 5 \mathrm{~cm}$ group } & \multirow[b]{2}{*}{ Refs. } \\
\hline & & Events $^{\mathrm{a}}$ & Total & Events $^{\mathrm{a}}$ & Total & \\
\hline Liu & 2007 & 30 & 38 & 13 & 37 & (7) \\
\hline Zuo & 2008 & 23 & 36 & 1 & 20 & (8) \\
\hline $\mathrm{Yu}$ & 2011 & 45 & 72 & 9 & 45 & (9) \\
\hline $\mathrm{Xu}$ & 2010 & 54 & 78 & 24 & 157 & (10) \\
\hline Vona et al & 2004 & 23 & 23 & 3 & 20 & (11) \\
\hline
\end{tabular}

${ }^{a}$ Events: number of cases with CTCs detected in the peripheral blood.

Table III. Association of circulating tumor cells (CTCs) level with I/II and III/IV tumor stage groups.

\begin{tabular}{|c|c|c|c|c|c|c|}
\hline \multirow[b]{2}{*}{ Study } & \multirow[b]{2}{*}{ Year } & \multicolumn{2}{|c|}{ I/II group } & \multicolumn{2}{|c|}{ III/IV group } & \multirow[b]{2}{*}{ Refs. } \\
\hline & & Events $^{\mathrm{a}}$ & Total & Events $^{\mathrm{a}}$ & Total & \\
\hline Liu & 2007 & 10 & 35 & 33 & 40 & (7) \\
\hline Zuo & 2008 & 3 & 17 & 21 & 39 & (8) \\
\hline Yu & 2011 & 9 & 54 & 45 & 72 & (9) \\
\hline $\mathrm{Xu}$ & 2010 & 18 & 139 & 60 & 96 & (10) \\
\hline Vona et al & 2004 & 3 & 13 & 23 & 30 & (11) \\
\hline
\end{tabular}

${ }^{a}$ Events: number of cases with CTCs detected in the peripheral blood.

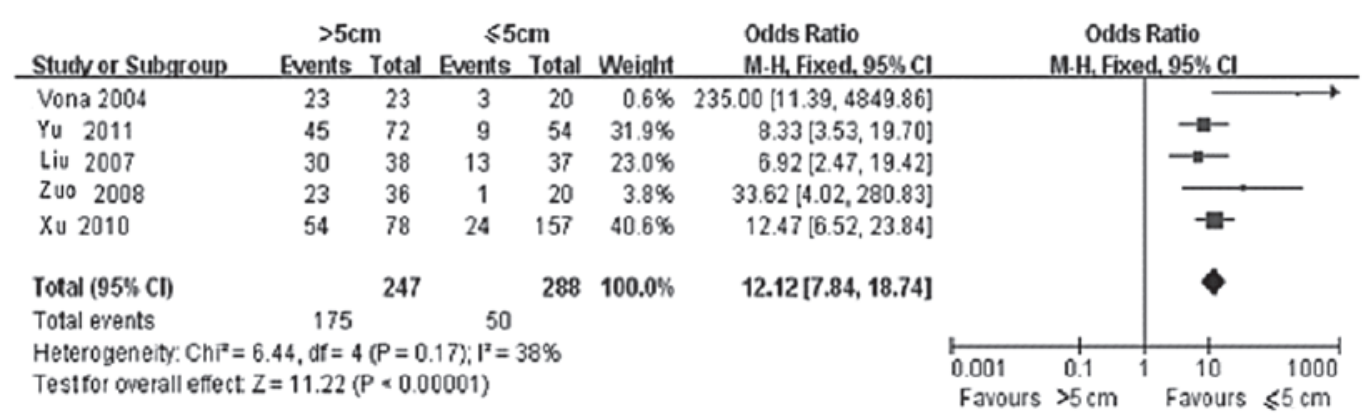

Figure 1. Comparison of circulating tumor cells-positive rate in the peripheral blood of the $>5$ and $\leq 5 \mathrm{~cm}$ tumor size groups.

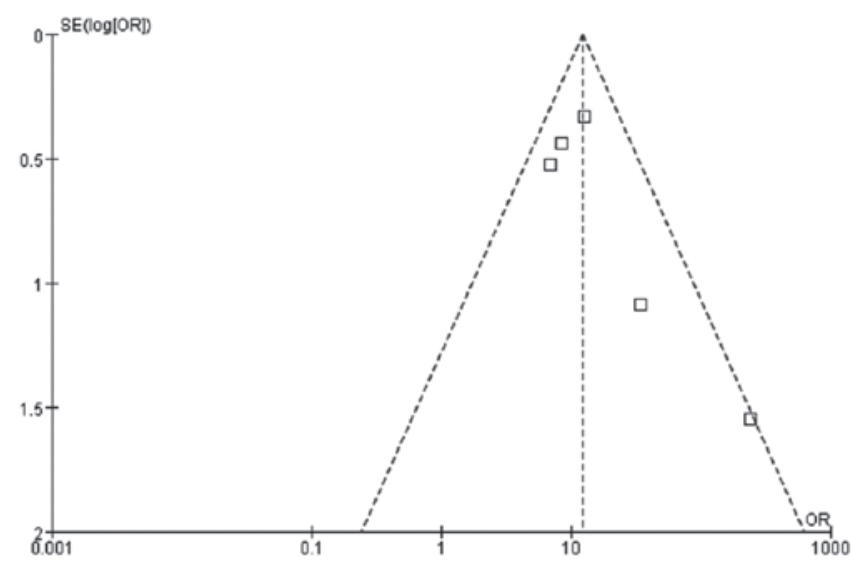

Figure 2. Funnel plot of the association between tumor size and circulating tumor cells-positive rate. 
Table IV. Association of circulating tumor cells (CTCs) level with the metastasis and non-metastasis groups.

\begin{tabular}{|c|c|c|c|c|c|c|}
\hline \multirow[b]{2}{*}{ Study } & \multirow[b]{2}{*}{ Year } & \multicolumn{2}{|c|}{ Non-metastasis group } & \multicolumn{2}{|c|}{ Metastasis group } & \multirow[b]{2}{*}{ Refs. } \\
\hline & & Events $^{\mathrm{a}}$ & Total & Events $^{\mathrm{a}}$ & Total & \\
\hline Liu & 2007 & 33 & 63 & 10 & 12 & (7) \\
\hline Zuo & 2008 & 1 & 20 & 23 & 36 & (8) \\
\hline $\mathrm{Yu}$ & 2011 & 40 & 110 & 14 & 16 & (9) \\
\hline $\mathrm{Xu}$ & 2010 & 11 & 121 & 67 & 114 & (10) \\
\hline Vona et al & 2004 & 6 & 16 & 20 & 27 & (11) \\
\hline
\end{tabular}

${ }^{\text {a} E v e n t s: ~ n u m b e r ~ o f ~ c a s e s ~ w i t h ~ C T C s ~ d e t e c t e d ~ i n ~ t h e ~ p e r i p h e r a l ~ b l o o d . ~}$

\begin{tabular}{|c|c|c|c|c|c|c|c|c|}
\hline Studv or Subgroup & \multicolumn{2}{|c|}{ I/ II } & \multicolumn{2}{|c|}{ III / N } & Weight & \multirow{2}{*}{$\begin{array}{l}\text { Odds Ratio } \\
\text { M.H. Fixed, } 95 \% \mathrm{Cl} \\
0.09[0.02,0.43]\end{array}$} & \multicolumn{2}{|c|}{$\begin{array}{c}\text { Odds Ratio } \\
\text { M.H. Fixed. } 95 \% \mathrm{Cl}\end{array}$} \\
\hline Yona 2004 & 3 & 13 & 23 & 30 & $7.8 \%$ & & & \\
\hline Yu 2011 & 9 & 54 & 45 & 72 & $23.4 \%$ & $0.12[0.05,0.28]$ & $=$ & \\
\hline Liu 2007 & 10 & 35 & 33 & 40 & $16.0 \%$ & $0.08[0.03,0.25]$ & & \\
\hline Zuo 2008 & 3 & 17 & 21 & 39 & $7.7 \%$ & $0.18[0.05,0.74]$ & & \\
\hline Xu 2010 & 18 & 139 & 60 & 96 & $45.1 \%$ & $0.09[0.05,0.17]$ & - & \\
\hline Total (95\% Cl) & & 258 & & 277 & $100.0 \%$ & $0.10[0.07,0.16]$ & & \\
\hline Total events & 43 & & 182 & & & & & \\
\hline $\begin{array}{l}\text { Heterogeneity. } \mathrm{Chi}^{2}= \\
\text { Test for overall effect }\end{array}$ & $\begin{array}{l}11, \mathrm{df}=4 \\
=10.43(\end{array}$ & $\begin{array}{l}(P=0 . \\
P=0.00\end{array}$ & $\begin{array}{l}89) ; F^{2}=0 \\
0001)\end{array}$ & & & & $\begin{array}{l}0.010 .1 \\
\text { Favours III / IV }\end{array}$ & $i_{\text {Favours I/II }}^{10} 100$ \\
\hline
\end{tabular}

Figure 3. Comparison of circulating tumor cells-positive rate in the peripheral blood in the I/II and III/IV tumor stage groups.

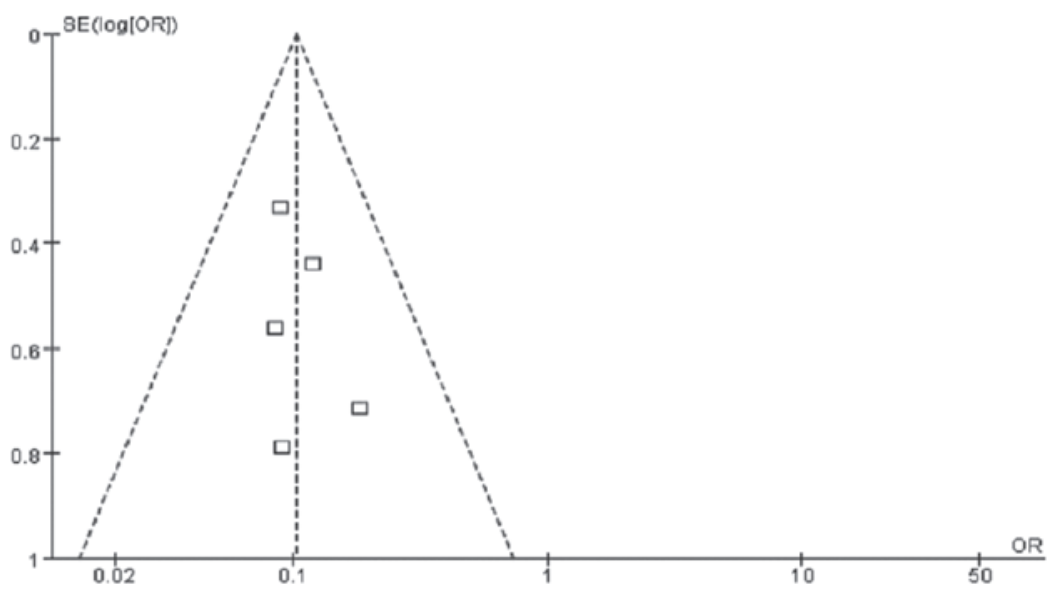

Figure 4. Funnel plot of the association between tumor stage and circulating tumor cells-positive rate.

statistical results of CTCs-positive rates in the two groups are presented in Table IV. Of the 5 clinical controlled trials, the non-metastasis group included 91 CTCs-positive cases, whereas the metastasis group included 134 CTCs-positive cases. According to the results of the $\mathrm{Q}$ test, no statistically significant heterogeneity was observed between the two groups $\left(\chi^{2}=4.29, \mathrm{DOF}=4, \mathrm{P}=0.37\right.$ and $\left.\mathrm{I}^{2}=7 \%\right)$; therefore, the fixed-effects model was applied. The difference in the CTCs-positive rate in the peripheral blood between the metastasis and the non-metastasis group was found to be statistically significant $(\mathrm{OR}=0.09,95 \% \mathrm{CI}$ : 0.05-0.15; $\mathrm{P}<0.00001)$ (Fig. 5). The comparison results revealed that the CTCs-positive rate in the peripheral blood in the metastasis group was significantly higher compared to that in the non-metastasis group. This finding indicates that the tumor metastasis was directly correlated with the presence of CTCs in the peripheral blood, i.e., patients with tumor metastasis exhibited a higher probability of CTCs detected in the peripheral blood. The funnel plot revealed no significant publication bias (Fig. 6). 


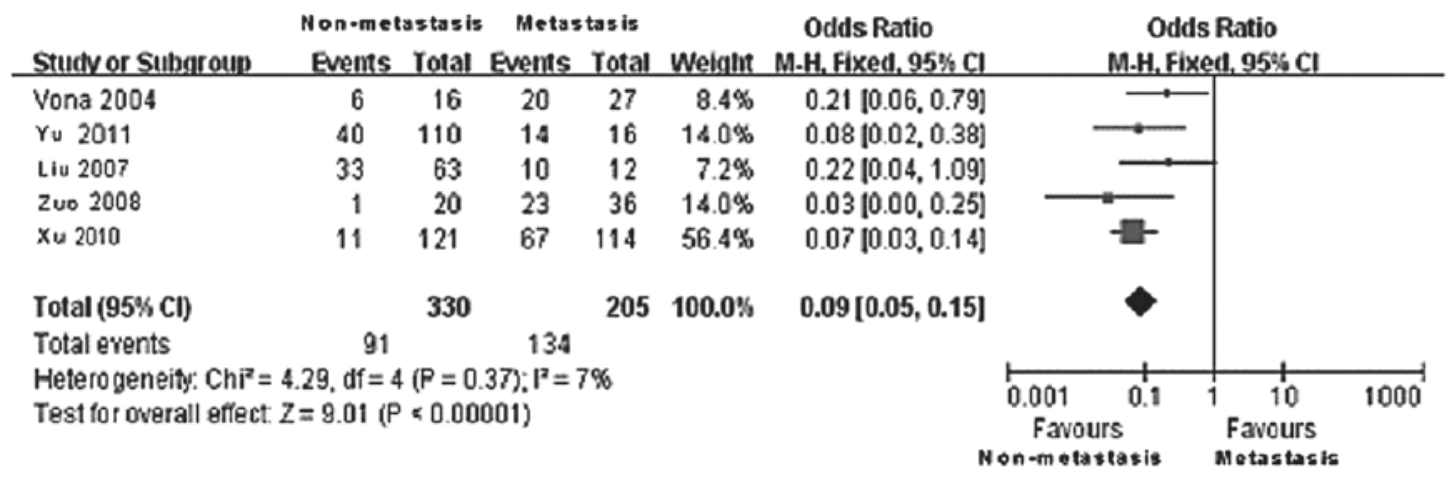

Figure 5. Comparison of circulating tumor cells-positive rate in the peripheral blood in the metastasis and non-metastasis groups.

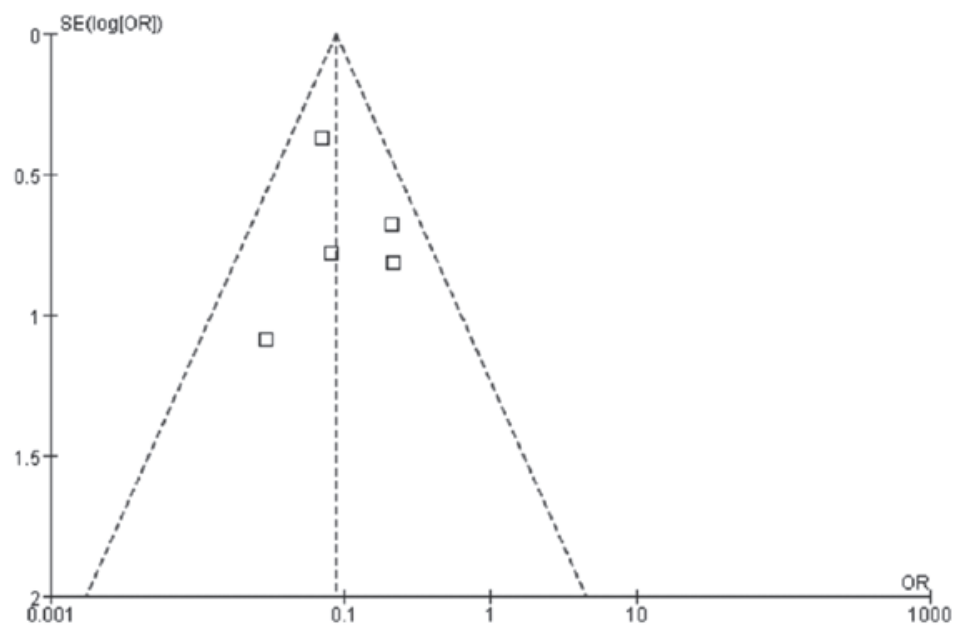

Figure 6. Funnel plot of the association between tumor metastasis and circulating tumor cells-positive rate.

\section{Discussion}

The diagnostic value of CTCs in liver cancer has been attracting increasing attention. This study aimed to summarize data from the literature published in China and other countries, conduct a meta-analysis using Review Manager 5.1 software and assess the correlation between CTCs level in the peripheral blood and tumor size, stage and metastasis. The results demonstrated that the CTCs-positive rate in the peripheral blood was directly correlated with tumor size, stage and metastasis. However, due to incomplete or missing data in the published studies, the effect of CTCs on tumor recurrence monitoring, prognosis, survival time and treatment customization could not be reviewed. However, considering the employment of CTCs in the diagnosis of malignant solid tumor, such as melanoma, breast, colorectal and prostate cancer, the clinical application of CTCs in liver cancer diagnosis may become more prominent with technological improvements (12).

CTC detection in the peripheral blood may be considered a viable alternative to cancer diagnosis. CTC detection assists in guiding molecular-targeted therapy and assessing anticancer efficacy. Of note: i) the development of a CTC detection method of high sensitivity and specificity is crucial for the follow up in clinical applications; ii) the investigations on novel CTC-specific markers may assist in improving the specificity and sensitivity of the identification and quantification of CTCs; iii) additional studies on the molecular and genetic constitution of CTCs may assist in elucidating the molecular mechanisms of cancer development, recurrence and metastasis; and iv) the role of cancer stem cells in tumor metastasis and drug resistance is being gradually emphasized. Follow-up studies in this field may assist in elucidating the mechanisms underlying tumor metastasis and may lead to the development of novel therapeutic interventions. Therefore, studies focusing on this area may promote advances in cancer biology and clinical cancer management, leading to improvement of the quality of life and prolongation of the lifespan of cancer patients.

\section{References}

1. Ashworth TR: A case of cancer in which cells similar to those in the tumors were seen in the blood after death. Aus Med J 14: 146-149, 1869.

2. Alemar $\mathbf{J}$ and Schuur ER: Progress in using circulating tumor cell information to improve metastatic breast cancer therapy. J Oncol 2013: 702732, 2013.

3. Ren C, Chen H, Han C, et al: Detection and molecular analysis of circulating tumor cells for early diagnosis of pancreatic cancer. Med Hypotheses 80: 833-836, 2013.

4. Andreopoulou E and Cristofanilli M: Circulating tumor cells as prognostic marker in metastatic breast cancer. Expert Rev Anticancer Ther 10: 171-177, 2010. 
5. Cohen SJ, Punt CJ, Iannotti N, et al: Relationship of circulating tumor cells to tumor response, progression-free survival, and overall survival in patients with metastatic colorectal cancer. Clin Oncol 26: 3213-3221, 2008.

6. Sun YF, Yang XR, Zhou J, Qiu SJ, Fan J and Xu Y: Circulating tumor cells: advances in detection methods, biological issues, and clinical relevance. J Cancer Res Clin Oncol 137: 1151-1173, 2011.

7. Liu D: Detection of circulating tumor cells in peripheral blood and its significance in patients with hepatocellular carcinoma (unpublished $\mathrm{PhD}$ thesis). Fudan University, 2007.

8. Zuo GH: Detection and biological characteristics of circulating tumor cells in peripheral blood of patients with liver cancer (unpublished PhD thesis). Third Military Med Univ, 2008.
9. Yu F: Improvement of circulating liver cancer cells detection and its preliminary study on the postoperative recurrence prediction (unpublished PhD thesis). Second Military Med Univ, 2011.

10. Xu W: Isolation/detection system and clinical application research of circulating liver cancer cells based on sialic acid glycoprotein receptor (unpublished PhD thesis). Second Military Med Univ, 2010.

11. Vona G, Estepa L, Béroud C, et al: Impact of cytomorphological detection of circulating tumor cells in patients with liver cancer. Hepatology 39: 792-797, 2004.

12. Ghossein R and Bhattacharya S: Molecular detection and characterization of circulating tumor cells and micrometastases in prostatic, urothelial, and renal cell carcinomas. Semin Surg Oncol 20: 304-311, 2001. 